\title{
Lumbatex: A Wearable Monitoring System Based on Inertial Sensors to Measure and Control the Lumbar Spine Motion
}

\author{
Juan M. Cortell-Tormo, Miguel García-Jaén, Daniel Ruiz-Fernández, Senior Member, IEEE
}

\author{
Vicent Fuster-Lloret
}

\begin{abstract}
To measure the curvature of the lumbar spine is an important challenge in disciplines related to Physical Therapy, Rehabilitation and Sports Medicine seeking to solve the incidence of the low back pain and other spinal disorders in population. In clinical practice, most of the methods used are manual or depend on the trained eye of the specialist who is measuring. We have developed Lumbatex: an integrated system based on inertial sensors integrated in a wearable textile device. This device is connected via Bluetooth to a software which interprets data from the sensors and provide real-time biofeedback to users in a graphical way and also a quantitative measure of the curvature and spinal motion. The system is tested in two ways: first, checking the accuracy detecting changes in curvatures; second, evaluating the usability and comfort from the user standpoint. The accuracy is checked through a static method getting curvature values from the device placed on curved platforms and a dynamic validation with volunteers performing different exercises. The results obtained showed a high accuracy measuring changes in curvature with an error lower than one degree in the static test, and a good usability and comfort according to the opinion of the volunteers.
\end{abstract}

Index Terms - spinal disorders; low back pain; lumbar spine; inertial sensors; medical rehabilitation; sports medicine.

\section{INTRODUCTION}

\section{A. Importance of Lumbar Spine Monitoring}

C SPINAL disorders include a broad spectrum of pathologies such as congenital, developmental, degenerative, traumatic, infectious, inflammatory, and neoplastic disorders and can encompass pain syndromes, disk degeneration, spondylosis, radiculopathy, stenosis, spondylolisthesis, fractures, tumors, and osteoporosis [1], [2]. Musculoskeletal disorders, including arthritis and lower back pain, are the second greatest cause of disability and they represent one of the greatest health problems in the world population [3]. The direct and indirect costs of treating spinal disorders are estimated at more than $\$ 100$ billion per year globally [4].

This study was supported by grant: DEP2011-30009-C02-01 from the Spanish Ministry of Economy and Competitiveness. Miguel García-Jaén participated in this study supported by a pre-doctoral grant ACIF/2016/048 from the Generalitat Valenciana, Spain. (Corresponding author: Daniel Ruiz Fernández.)

J. M. Cortell-Tormo, M. García-Jaén, and V. Fuster-Lloret are with Area of Physical Education and Sports, University of Alicante, 03690 San Vicente del Raspeig, Spain

D. Ruiz-Fernández is with Department of Computer Technology, University of Alicante, 03690 San Vicente del Raspeig, Spain.
In this sense, in a recent systematic review about prevalence of low back pain (LBP), authors explained that LBP is a very common and costly musculoskeletal disorder worldwide. In fact, the number of individuals with lower back pain will tend to increase over the next decades due to the sedentary lifestyle of modern societies [5]. Thus, non-specific LBP is a major public health problem worldwide with a lifetime prevalence of $84 \%$ [6] and it is associated with decreased work ability and, consequently, increased risk of long-term sickness absence among working age adults [7].

LBP can be treated by active (exercise, education, prevention, and multimodal therapies) or passive therapies (physical modalities, manual therapies, reflex therapies, assistive devices, and drugs) [8], [9]. Although it is possible to find many studies using different therapies to treat this spinal disorder, both spinal therapeutic exercises [10] and a constant postural control [11] are the strategies widely accepted as good methods to reduce the LBP incidence. Physical therapies through exercises play a major role in the processes of prevention, pain reduction, and rehabilitation of LBP [12] [15]. Research indicates that rehabilitation and prevention exercise programs, most commonly used as treatments for patients with chronic LBP, are based on trunk strengthening exercises and trunk stabilization exercises [10], [15].

Stabilizing the lumbar spine through the control of the neutral zone of lumbar motion (i.e., preserving normal low back curve similar to that of upright standing) and avoiding full lumbar flexion positions seems to be a relevant factor in intervention programs through exercises for the low back. This would provide protection from ligament injury and posterior disc herniation [16] - [19]. Therefore, suitable exercises are those in which the lumbo-pelvic region remains aligned within this neutral zone whereas the subjects perform movements using their extremities [17] - [19]. In this sense, the current trend is to train through functional exercises simulating movements that have a close relation with professional and recreational physical demands. That is to say, performing functional multiplanar movements while maintaining the lumbar spine stable and aligned. Hence, it has been found that training based on global and multiplanar controlled movements has positive effects for the prevention and treatment of LBP - if the neutral zone of the lumbar spine is specifically monitored [16] - [21].

Monitoring, quantification and measurement on lumbar 
spine position and motion is commonly used within the clinical setting. It is used to assess a lumbar lesion or pathology, to diagnose LBP and less frequently to treat it [22]. Nevertheless, most of the clinical techniques commonly used, such as skin distension, fingertip-to-floor and inclinometers are unable to provide sufficient information about spinal movement patterns since they comprise measurements in only one or two dimensions. However, it is noticeable that the lumbar motion presents a complexity of movements in the three planes of motion (sagittal, coronal and transverse planes) [23], [24]. In contrast, some laboratory methods, such as stereoradiography, electromagnetic or opto-electronic three-dimensional videobased systems can provide this extra information. Nonetheless, the reporting data of these methods often have been only limited to the assessment of the range of motion (ROM). Additionally, they are too complex, time consuming and expensive methods [23] - [25]. These drawbacks restrict these methods to a laboratory environment with experienced staff and consequently, make them unsuitable for a routine clinical and rehabilitative or preventive use [23] - [25].

However, in a clinical, rehabilitative or preventive exercise settings, when performing global and multiplanar spinal movements, the monitoring of lumbar spine position should be essential. Therefore, its measurement and monitoring should be mandatory, although it is difficult and challenging [20], [21]. In fact, defects unnoticed in the rachis position can generate unnecessary tensions in the spine with antagonistic results to those that motivate the purpose of a specific exercise. For this reason, experts agree on the importance of the external monitoring and feedback in the correct technical execution of these exercises and spinal movements [20], [21], [26] - [28]. It is also possible to use different subjective methods, such as visual correction, verbal instructions and manual assistance during the development of certain exercises or physical activities in order to monitor changes in the lumbar spine. However, their main limiting agent is that the only benchmark would be the observation of the specialist. Often a technical evidence to accurately confirm postural information in an objective way would be needed. With the recent development in Micro-Electro-Mechanical Systems (MEMS) sensor technology, wearable inertial sensors have been proposed to overcome the main limitations of these subjective methods, as well as laboratory methods. Inertial sensors are simple to use and commercially available, their price is substantially lower, and they enable field-based accurate measurement. All that allows them to be used in different areas, such as clinical, rehabilitative or preventive and training sports settings [25], [29] - [32].

\section{B. Spine Movement Monitoring Systems}

It is possible to find spine movement monitoring systems in previous research that help specialists both providing feedback of the back posture or determining the shape and morphology of the spine [33], [34] - [39]. These are optoelectronic-based systems, motion capture systems or computer-assisted electromechanical devices. They comprise the use of accelerometers, electronic goniometers, and strain-gauge based technology. The main problem with most of these devices is the low flexibility and the limitation of having the patients in a controlled environment. This reduces, many times, their mobility and evaluates movements in non-real situations or uncommon movements. The consequence of performing back movements with these monitoring limitations is that it is difficult to be sure how these rehabilitation exercises will work in patients' daily-life. Meanwhile, how suitable they will be for some postural tasks in training exercises for athletes.

On the other hand, in recent years, devices based on pressure and strength have been also introduced but they have also significant limitations on its use. These instruments are valid for detecting changes of spinal curvature through changes in the pressure unit produced by lumbar movement. They are commonly used in laboratory or rehabilitation and physical therapy settings to control that no shifts on lumbar lordosis occurring while it is performing certain lumbar stabilization maneuvers (like abdominal drawing-in maneuver). However, these devices cannot measure the degree of lumbar curvature and its changes directly. Only from this variation on pressure, they can indirectly estimate changes on lumbar curvature. Consequently, the direct monitoring of lumbar spine kinematics using these pressure-based systems would have a high margin of error, thereby limiting their accuracy when working with these instruments in these settings. Additionally, all these devices have as major drawback that they do not estimate changes in lateral bending and rotation. It is increasingly valued when control exercises and/or therapies require knowledge of aspects related to these planes of movement.

Some examples of patents protecting such systems are presented. Patent US5338276 entitled Exercise monitoring device was developed to monitor changes in the lumbar area from a change of pressure. Moreover, this device needs support points which leads to an important additional limitation when it is applied in certain exercises or therapies because several important rehabilitation exercises and maneuver are performed in standing, prone or sitting position without support points. The patent NL1023363 entitled Incorrect posture device detection comprises pressure sensor mounted on a stiff part attached to a belt and connected to a warning device. It assesses changes in the rachis from a pressure mechanism located in the middle of the lumbar spine.

Other devices such as those included in the patent US5402107 entitled Apparatus for sensing body attitude and US5398697 entitled Apparatus for monitoring spinal motion, present an improbable instrumentation. Specifically, the patent US5398697 uses as a monitor element a light beam which makes impossible to quantify how many changes occur in the spine position. In consequence, the characteristics of this device make it very difficult to use in supine positions.

The AU2005247045 patent entitled Apparatus and method for monitoring strain and / or load applied to a mammal assesses changes in the spinal column from two accelerometer sensors and two surface electromyography sensors. From neuromuscular activation and changes in space accelerometers, it estimates the curvature and changes in the lumbar spine. The drawback of this device is that using only two sensors, placed 
in L1 and S2, it calculates the lumbar curvature, but it has an important limitation to provide direct information on the degree of involvement of each of the vertebral segments in the formation of such lumbar lordosis. Furthermore, it is possible that the information about changes in tilt and rotation calculated from sensors located in the central area of the trunk could limit the accuracy of this device, especially detecting asymmetries. These aspects are determining factors when the therapies are monitored.

Finally, other important issue that is not considered in all the systems previously analyzed is the usability of the device and its user's comfort degree out of a controlled environment. It is important that a person could carry the device easily joining usability and accuracy when data are collected.

Therefore, there is a need to develop new devices that overcome the aforementioned limitations and provide accurate information about postural feedback and segmental motion of the lumbar spine. In this sense, to measure and quantify the spinal movement using inertial sensors in a wearable device could make it intuitive and user-friendly for the final user of this system. In fact, wearable inertial sensors have already been used to measure the range of motion of spine in previous studies because their data can be easily transformed into useful parameters that enable a real-time spinal motion measurement system [40], [41].

Therefore, the main objective of this research is to develop a wearable system based on smart textiles that allows to quantify the segmental position of lumbar curvature. This device would also allow to monitor and measure the movements and changes in the lordosis of lumbar spine in a sagittal plane. Together with this device, we will develop a software that, via Bluetooth, obtains the data from the sensors and it interprets the data showing the results in a graphical way. Finally, using a visual analogue scale, we will analyze the user's comfort degree of this device. Thus, the main aims of this study are to carry out the mechanical and dynamic validation of the system and the usability and comfort assessment of the belt.

\section{Materials AND MethodS}

\section{A. System Development}

The proposed system consists of two main parts: The proposed system consists of two main parts: a set of sensors attached to a textile belt with Velcro-fastening, for the detection of the position and movements in the lumbar spine, and the software and Bluetooth receptor of the data. There are seven sensors in the system connected with a control circuit, as can be seen in the Fig. 1. The system uses five sensors in the central area detecting the degree of lordosis lumbar and flexionextension movements of lumbar spine, which match to the lumbar vertebrae L1-L5. These sensors are directly attached to the skin of the person in each anatomical reference from each lumbar spinous process using a dermatological adhesive tape $\left(3 \mathrm{M}^{\mathrm{TM}}\right.$ Double Coated Medical Tape). Furthermore, there is another sensor in each side of the spine in order to detect rotation and lateral bending movements of the lower back. Sensors uses a combined 3-axis accelerometers and gyroscopes in the same device. This way not only we avoid problems related to pressure sensors but also, we improve the accuracy of movements' detection. Apart from the sensors, there are also the necessary electronics to get the data from the sensors and to analyze them. Finally, we store all the electronics in a slim box located outside the belt. The box is $10 \mathrm{~cm}$ length, $6 \mathrm{~cm}$ width and $2 \mathrm{~cm}$ depth. The weight of the system (included the textile) is 150 gr (Fig. 1).

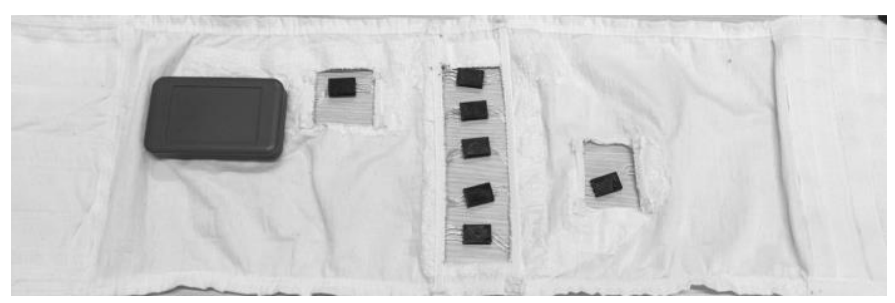

Fig. 1. Textile integrated sensor system

The architecture of the system can be divided in four main blocks, which are associated with the specifics needs of energy (power block), information acquisition (sensor block), processing (core of the system) and the external interaction (communications block). We can check the different integrated blocks in the electronics of the system in Fig. 2. Additionally, there is an essential element of the system: the software which allows the correct configuration of the sensors as well as the correct visualization of the detected curves segmentally. Finally, a small box with brackets contains the power, communications and processing blocks. This way, the blocks are joined to the belt and connected to the sensors (Fig. 3). In addition, this box incorporates a switch to turn on and off the system, a USB connector for charging and connecting the box and two LEDs indicating if the device is on or off and charging or not the battery.

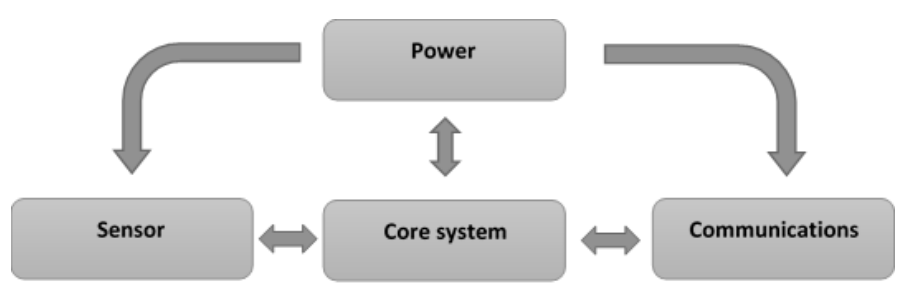

Fig. 2. Diagram of blocks of the electronics board.

\section{1) Power Block}

We can supply energy to the system using non-rechargeable batteries or through a little rechargeable battery. The option of using a rechargeable battery implies to add the necessary electronics for its management together with the connectors for the charge. Although there are extra components, we think that the best option is the rechargeable battery. Thus, we used Li-Po rechargeable batteries and a mini USB type B plug to recharge them. USB socket is just used for battery charging. All the communication with the device is wireless, through Bluetooth technology. The autonomy depends on the use of the system; usually between four and six hours.

\section{2) Sensor Block}

We incorporate 3-axis accelerometers and gyroscopes to register the position, movements and changes of the lumbar 
spine in the sensor block. The selected sensor has been the MPU6000 from Invensense $\odot$ that includes accelerometer and gyroscope. In the electronics board designed, there are 21 digital I/O and 8 analogic inputs. We use the digital I/O to connect several peripherals, such as timers, UART, ADC, etc. Fig. 1 shows the location of the sensors in the device. Five of the sensors are in a straight line to be placed on top of each lumbar spinous process; a sixth sensor is placed in the upper right part of the belt; finally, the seventh sensor is placed in the lower left part of the belt. Sensors are connected to the central PCB through brackets and wires knitted in the belt (in Fig. 1, sensors can be observed, and wires are hidden to avoid problems when the belt is worn). Wires are used both for data and power. Fig. 3 shows the brackets and the size of the box with brackets.

\section{3) Core System}

In the core system we include the microcontroller CC2540256K of Texas Instruments, which is based on 8051 architecture. Apart from the microcontroller, we use a $32 \mathrm{Mbits}$ chip of low consumption flash memory with SPI interface. The real-time clock (RTC) used in the device is also in this block. The RTC has also a SPI interface and a backup battery to support time and date.

\section{4) Communications Block}

Finally, in the communications block we find the necessary hardware for the Bluetooth communication. There are several options for the antenna (on-chip, external, on-PCB) and we choose a PCB antenna that accomplishes BLE (Bluetooth Low Energy) specifications with $8-10$ meters range and omnidirectional.

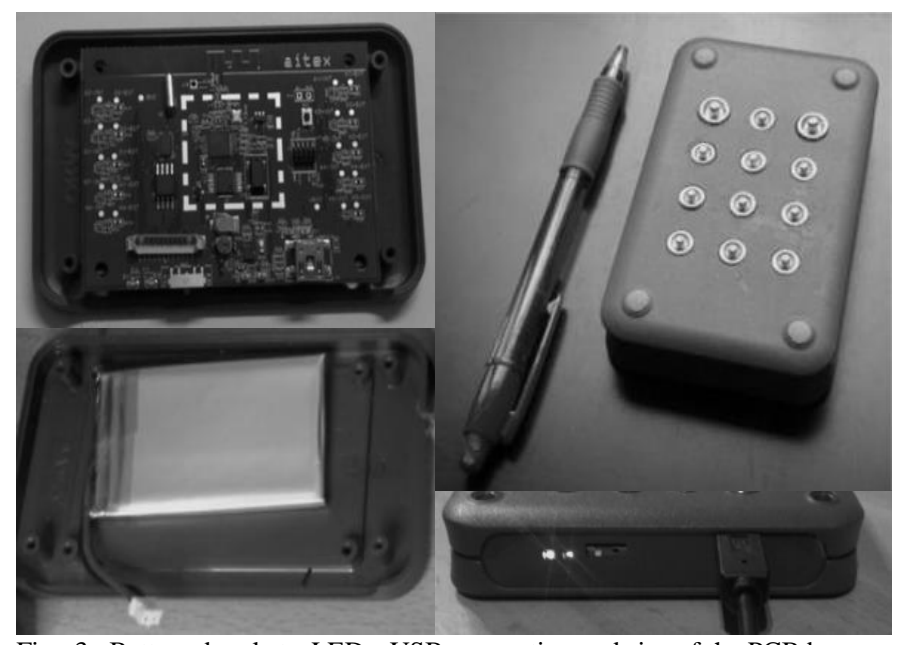

Fig. 3. Battery, brackets, LEDs, USB connection and size of the PCB box.

\section{5) Software}

Other fundamental part of the system is the software, designed as a server to which the control hardware can connect through BLE. The software runs on a Windows@ operating system and it allows a total control over the sensors (connecting, disconnecting, accessing to data), stores the data (making a $\log$ ) and draws the movements in different axis. We also develop a software for mobile devices that runs on ANDROID operating system (higher than 4.0) and with BLE.
Fig.4 shows some screenshots of the desktop software and the application for mobile devices. This software presents the data both numerically and visually to facilitate its interpretation. Moreover, it generates absolute and relative data allowing a better information of the evaluated reality. On the other hand, the software also reports the contribution degree of each one of the sensors in the generated curve. Thus, we can assess the degree of participation of each of the segments of the lumbar spine in the resulting curve. This aspect is essential for rehabilitation and physical therapy specialists. The design incorporates the graphic representation and the defined data for the values of absolute curvature for the $\mathrm{XY}, \mathrm{XZ}$ and $\mathrm{YZ}$ planes, the values of relative curvature for the $\mathrm{XZ}$ plane, the percentage values of contribution of each segment $L$ to the final value (sagittal plane) and the difference between the lateral sensors for measuring lateral bending and rotation.

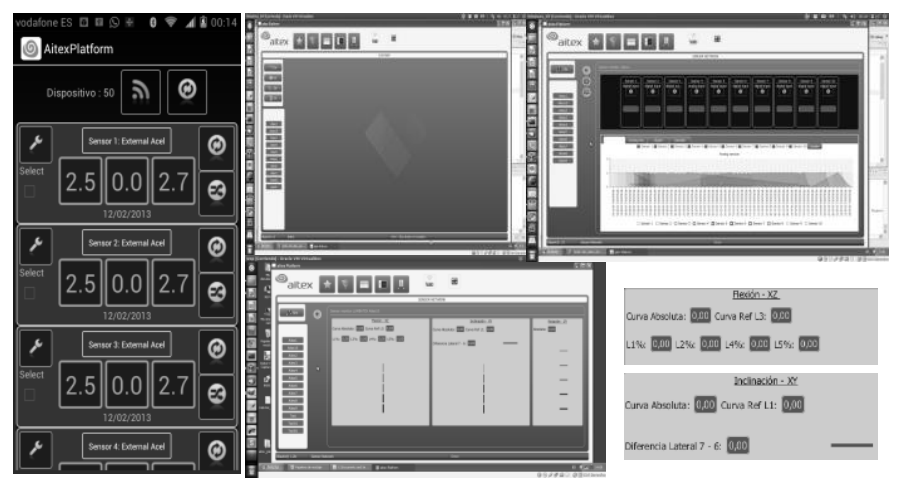

Fig. 4. Screenshots of the developed software.

\section{6) Coordinate System}

In order to obtain the angles from the coordinates given by each accelerometer, we use the tangent arc function and the acceleration measures of the sensors, which are sent to the server with $1 \mathrm{~Hz}$ frequency. The axis is taken according with the specifications of the MPU6000 sensor shown in Fig. 5.
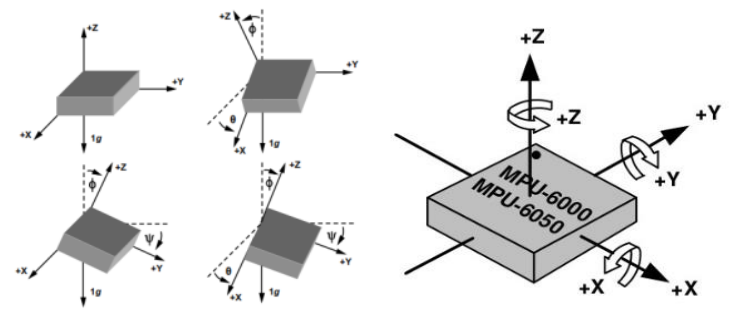

Fig. 5. Coordinate system: Axis for the MPU6000 sensor.

The accelerometers of the sensors are configured in such a way that the sensor is located on the $\mathrm{Z}$ axis, which is perpendicular to the lumbar area and the $\mathrm{Y}$ axis, which is aligned with the spine. Each accelerometer has its own individual coordinate system although they match in the distribution of the axes. The measuring unit is internal to the accelerometer and is expressed in steps, which indicate the acceleration in each of the axes. Specifically, the accelerometers are configured for $2 \mathrm{G}$ (being $1 \mathrm{G}$ equivalent to 16384 steps). 
The method used in this study for inclination detection with three axes is based on the determination of the individual angle for each axis of the accelerometer from an absolute reference position. The reference position is taken as the typical orientation of a device with the axes $\mathrm{X}$ and $\mathrm{Y}$ in the plane of the horizon ( $0 \mathrm{G}$ of gravitational field) and the $\mathrm{Z}$ axis orthogonal to the horizon ( $1 \mathrm{G}$ of gravitational field). In the initial position of $0 \mathrm{G}$ on the $\mathrm{X}$ and $\mathrm{Y}$ axes and $1 \mathrm{G}$ on the $\mathrm{Z}$ axis, all calculated angles would be 0 degrees. Using trigonometry, an angle $\varphi$ from the three acceleration values can be calculated as:

$$
\varphi=\tan ^{-1}\left\{\frac{a_{y}}{\sqrt{a_{x}^{2}+a_{z}^{2}}}\right\}
$$

Where $a$ is the acceleration value in $\mathrm{m} / \mathrm{s}^{2}$ obtained after converting the value of digital steps in which each accelerometer is calibrated to an analogue acceleration amplitude value.

\section{B. Testing Methodology}

\section{1) Mechanical Validation of Lumbatex}

Firstly, we decided to evaluate the reliability of the device by using artificial pieces simulating different degrees of spinal curvature. For that purpose, we designed six platforms with curves of 10, 15, 16, 17, 20 and 25 degrees. Then, we compared the measures taken with the system and the real curvatures. That way we studied the precision in platforms with 1 degree of difference $(15,16,17)$ and we considered a minimum of 10 degrees (we could not identify a lumbar problem with a lower angle) and a maximum of 25 degrees (the patient should follow a different treatment, not just physical therapy, with an upper angle). Subsequently, the device was individually tested for each curvature placing the five adhesive tapes along the piece with a separation of $3 \mathrm{~cm}$ (corresponding to a regular distance between each lumbar spinous process) and making the two extremes match with each end of the piece (Fig. 6). Then, each one of the five central sensors were fixed in the piece placing the belt vertically on a support that allowed it to remain immobile during the measurements. Finally, the data were stored for 30 seconds with a frequency of $1 \mathrm{~Hz}$ and they were exported to a spreadsheet for the conversion of the accelerometry data into angular values and, from these, to determine the corresponding curve. We repeated this experimental procedure 12 times for each piece.

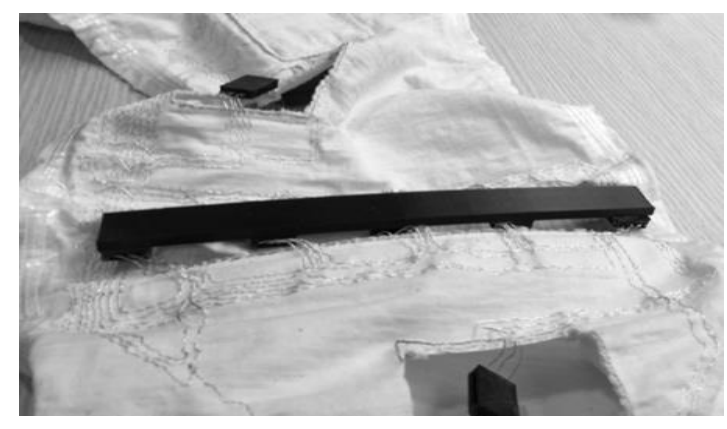

Figure 6. Platform with sensors
With the data in angular values, we calculated the descriptive statistics (mean, standard deviation, standard error, range and variance) for each variable. All the variables presented a normal distribution (Shapiro-Wilk test) and the variances were homogeneous (Levene's test). To identify the absolute variability, we calculated the mean standard error (MSE) and the coefficient of variation $(\mathrm{CoV})$ intra-device for each sensor and for the 12 measures analyzed in each condition. The confidence limit was calculated using the following formula: above $95 \%$ Limit $=\overline{\mathrm{x}}+(\mathrm{SE} \times 1.96)$ and below $95 \%$ Limit $=\overline{\mathrm{x}}-$ $(\mathrm{SE} \times 1.96)$. Relative reliability was estimated using the Intraclass Correlation Coefficient (ICC). The confidence limit was calculated at $95 \%$. An ICC of 0.9 was considered as very high, between 0.7 and 0.89 high, from 0.5 to 0.69 moderate and below 0.5 low. Statistical analysis was performed using the statistical package SPSS v.22 (SPSS Inc., Chicago, IL, USA).

Finally, the different curves were firstly traced on paper and after scanned in order to make the visual comparison of the similarity degree between the real curves and the generated curves from the device. After that, 6 circumferences and their corresponding radii were drawn. Then, they were sized to obtain a ratio of height and width from 1 to 1 . In order to perform the visual comparison with the results of overall accelerometers, the curves resulting from the measurements of the device were exported to the specific technical design software. At last, we could perform the visual comparison thanks to the circumferences represented from the calculated radius and the experimentally obtained curves represented with the same proportion and aspect ratio.

\section{2) Dynamic Validation of Lumbatex}

A convenience sample of 15 healthy volunteers (10 men and 5 women), recruited from a population of students at the University of Alicante, participated in this experimental protocol for the dynamic validation of the device (mean $\pm S D$ : age, $22.27 \pm 3.305$ years; body mass, $66.52 \pm 10.44 \mathrm{~kg}$; height, $171.9 \pm 10.21 \mathrm{~cm})$. Inclusion criteria included no history of pain in the low back and to know how to perform all the experimental conditions of the tests correctly. All participants provided written informed consent to participate in this study, which was approved by the University.

Three lumbar stabilization tests were planned for the dynamic reliability of the belt: corset test in prone position; corset test in modified prone position with anterior pelvic tilt; corset test in modified prone position with posterior pelvic tilt. To correctly perform the tests, participants had to correctly control the drawing-in manoeuvre, which causes the contraction of Transversus Abdominis muscle without moving the lumbo-pelvic complex (42). Then, each participant, with the Lumbatex belt fastened, had to lie down on a stretcher in prone position. Using the Stabilizer ${ }^{\circledR}$ Pressure Biofeedback Unit (PBU) at an initial pressure of $70 \mathrm{mmHg}$ for each test, each participant was asked to carry out the different tests during a 30 -second period of data collection. The PBU pressure was reduced or enhanced in $10-20 \mathrm{mmHg}$ depending on each test as indicative to a correct performance of this exercise. The pelvic tilt and the drawing-in manoeuvre modified the pressure in the $\mathrm{PBU}$, and the belt registered the lumbar curvature at all times of the test. 
We applied the Pearson correlation coefficient $(r)$ for each study condition in order to calculate the relationship between the data of the PBU and the data provided by the belt. For all the tests, we selected the central data of the measurement derived from the belt while we collected the $\mathrm{PBU} \mathrm{mmHg}$ values every second of the measurement. The analysis of mean variance between the three study conditions was carried out applying an ANOVA test and the post hoc DMS test. The level of significance was established in $p<0.05$ for the analysis of variance and $p<0.01$ for the correlation test. Statistical analysis was performed using the statistical package SPSS v.22 (SPSS Inc., Chicago, IL, USA).

\section{3) Usability and Comfort Assessment of Lumbatex}

The same sample of volunteers described in the previous point participated in the usability and comfort assessment of the device filling in an adapted comfort questionnaire. Thus, the analysis of the perception of comfort was performed using the $150 \mathrm{~mm}$ analogue visual scale (43). This scale consists of a horizontal line of $15 \mathrm{~cm}$ long. The limit on the left represents the "not comfortable" perception and the limit on the right the "most imaginable comfortability". The participants were asked to perform several postures and exercises with or without movement in the lumbo-pelvic region. Afterwards, they filled in the beforementioned comfort questionnaire marking the scale based on their subjective perception of usability and comfort of the device. The items evaluated were: waist and spine fastening, tissue compression, static and dynamic usability and comfort, temperature, humidity, design and general perception of comfort of the belt. The obtained descriptive data and their percentage values were analyzed for each of the items evaluated.

\section{RESULTS}

\section{A. Results for the Mechanical Validation of Lumbatex}

First, it has been tested the mechanic and technic reliability of the device. The next section shows the results for one of the study conditions: the $25^{\circ}$ curve. Similar results were obtained for the other curves.

\section{1) Results for the $25^{\circ}$ Curve Condition}

The values corresponding to the descriptive statistics for each of the five sensors in the $25^{\circ}$ condition are shown in the Table I. The absolute reliability obtained is very good in all the sensors.

TABLE I

DESCRIPTIVE STATISTICS FOR EACH SENSOR IN THE $25^{\circ}$ CURVE CONDITION

\begin{tabular}{|c|c|c|c|c|c|c|c|}
\hline Sens & Min & Max & $\begin{array}{r}\quad(\mathrm{N}=? \\
\text { Mean }\end{array}$ & 20) & MSE & $\begin{array}{c}95 \% \\
\text { CL }\end{array}$ & Var \\
\hline 1 & 100.486 & 101.471 & 100.773 & 0.087 & 0.004 & $\begin{array}{c}100.766- \\
100.781\end{array}$ & 0.008 \\
\hline 2 & 100.323 & 100.730 & 100.505 & 0.072 & 0.003 & $\begin{array}{c}100.499- \\
100.511\end{array}$ & 0.005 \\
\hline 3 & 91.856 & 93.561 & 92.586 & 0.095 & 0.004 & $\begin{array}{c}92.578- \\
92.594\end{array}$ & 0.009 \\
\hline 4 & 84.554 & 85.063 & 84.775 & 0.083 & 0.004 & $\begin{array}{c}84.768- \\
84, .82\end{array}$ & 0.007 \\
\hline 5 & 82.778 & 83.707 & 83.104 & 0.091 & 0.004 & $\begin{array}{l}83.003- \\
83.018\end{array}$ & 0.008 \\
\hline
\end{tabular}

Sens=Sensor; Min=Minimum; Max=Maximum; SD=Standard Deviation; MSE=Mean Standard Error; 95\%CL=Confidence Limit; Var=Variance

The graphic combination of the angles generated by each one of the sensors allows to generate a coherent form with the $25^{\circ}$ condition, extracting the average data of all the measurements.

The Intraclass Correlation Coefficient (ICC) for the $25^{\circ}$ condition showed the values in the Table II. For all the sensors $\mathrm{p}$ is $<0.001$. The relative reliability shows very high values (ICC>0.9) in all the sensors.

TABLE II

ICC VALUES FOR THE $25^{\circ}$ CURVE CONDITION

\begin{tabular}{ccc} 
Sensor & ICC & $\mathbf{9 5 \%}$ CI \\
\hline $\mathbf{1}$ & 0.980 & $0.969-0.988$ \\
$\mathbf{2}$ & 0.994 & $0.991-0.996$ \\
$\mathbf{3}$ & 0.968 & $0.951-0.981$ \\
$\mathbf{4}$ & 0.995 & $0.992-0.999$ \\
$\mathbf{5}$ & 0.976 & $0.962-0.986$ \\
\hline
\end{tabular}

\section{2) Comparison of the Similarity Degree between the Real Curves and the Generated Curves from Lumbatex}

Fig. 7 shows an overlap of the mathematically generated standard curves and those curves generated by the device from all measured conditions. Each black color curve has been generated by the device and the black color curve is the standard curve. As it can be seen, if the mathematically generated standard curve is placed on the same surface and the curve generated by the device is superimposed on it. A high degree of similarity can be observed between them.

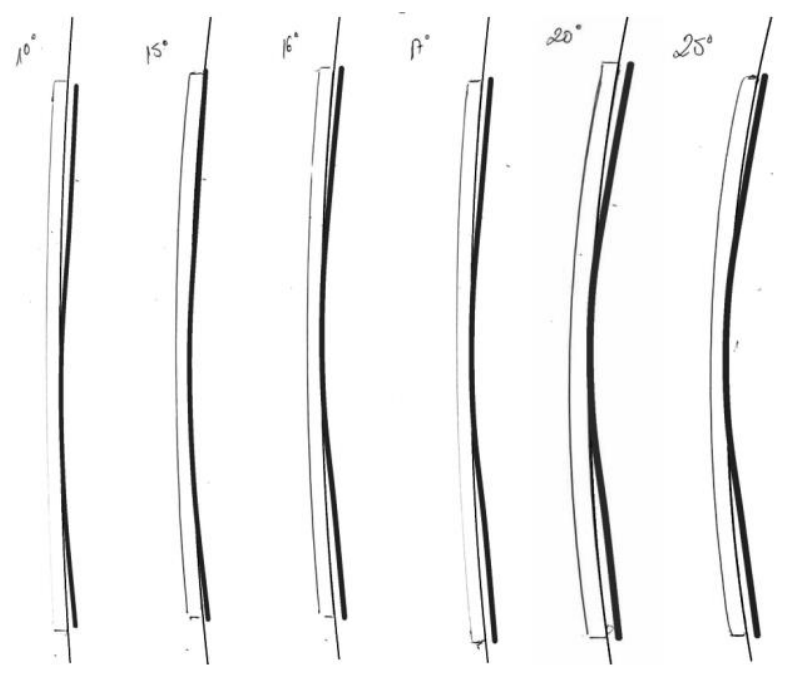

Fig. 7. Superposition of all measured conditions with standard curves.

In the Table III, it is shown the mean of the degree values obtained measuring the curves provided by the device for each curvature. The curvature degree has been got from the curvature radius, obtained through geometry functions from three points 
in the curvature, the sensor one, five and the centre, sensor three. Although the curvatures are not exact the important question to remark is that the evolution showed in the curvatures measured is useful enough to detect the changes on different generated curvatures. This would be the main purpose of the Lumbatex system.

\section{TABLE III}

CURVATURES COMPARISON BETWEEN THE CURVES FROM THE LUMBATEX AND THOSE ARTIFICIALLY GENERATED

\begin{tabular}{cccc}
$\begin{array}{c}\text { Artificial } \\
\text { curve } \\
\text { (degrees) }\end{array}$ & $\begin{array}{c}\text { Mean of the } \\
\text { Lumbatex } \\
\text { curves (degrees) }\end{array}$ & $\begin{array}{c}\text { Standard } \\
\text { deviation }\end{array}$ & $\begin{array}{c}\text { Curvature } \\
\text { difference } \\
\text { (degrees) }\end{array}$ \\
\hline $\mathbf{1 0}$ & 9.1868 & 0.3729 & 0.8132 \\
$\mathbf{1 5}$ & 15.6177 & 0.4683 & 0.6177 \\
$\mathbf{1 6}$ & 16.1792 & 0.3583 & 0.1792 \\
$\mathbf{1 7}$ & 17.1198 & 0.3886 & 0.1198 \\
$\mathbf{2 0}$ & 19.2734 & 0.473 & 0.7266 \\
$\mathbf{2 5}$ & 24.3475 & 0.4297 & 0.6525
\end{tabular}

\section{B. Results for the Dynamic Validation of Lumbatex}

Secondly, it has been tested the dynamic reliability of the device. Next sections show the results regarding the relationship between the data of the PBU and the data from the belt and also the results regarding the estimated variance for each experimental condition. Finally, a visual graphic representation generated from the measured data by the device in the three experimental conditions are shown in the last subsection.

\section{1) Results Corresponding to the Relationship between the $P B U$ and Lumbatex Data}

Table IV shows the results of the correlations between the data provided by the PBU and the data generated by the device for each of the three experimental tests.

TABLE IV

PEARSON'S CORRELATION COEFFICIENTS BETWEEN PBU AND LUMBATEX FOR EACH VARIATION OF THE CORSET TEST

\begin{tabular}{|c|c|c|c|c|c|c|}
\hline & & S1 & S2 & S3 & S4 & S5 \\
\hline PBU & $\begin{array}{c}\text { Corset } \\
\text { Test }\end{array}$ & $0.861 *$ & $0.953 *$ & $0.943 *$ & $0.767^{*}$ & $0.832 *$ \\
\hline PBU & $\begin{array}{c}\text { Corset } \\
\text { Test } \\
\text { [Anterior } \\
\text { Pelvic } \\
\text { Tilt] } \\
\end{array}$ & $0.986^{*}$ & $0.978 *$ & $0.964 *$ & $0.959 *$ & $0.931 *$ \\
\hline PBU & $\begin{array}{c}\text { Corset } \\
\text { Test } \\
\text { [Post. } \\
\text { Pelvic } \\
\text { Tilt] } \\
\end{array}$ & $0.914 *$ & $0.954 *$ & $0.940^{*}$ & $0.962 *$ & $0.956^{*}$ \\
\hline
\end{tabular}

$\mathrm{PBU}=$ Pressure Biofeedback Unit; $\mathrm{S} 1,2 \ldots=$ Sensor $1,2 \ldots$

*The correlation is significant at the level 0.01 (bilateral)

For the Lumbatex, the values have been estimated from the average values of each of the tests and for each sensor. Then, the percentage of change for each test and sensor in the other two tests has been estimated from the corset test in prone position as a reference. Finally, the percentages of each test have been added. For the PBU data, from the initial value of 70 $\mathrm{mmHg}$, the percentage of change in the modified tests has been calculated regarding to the initial value of the first test (see Fig. 8).

\section{-PBU $=$ Lumbatex}

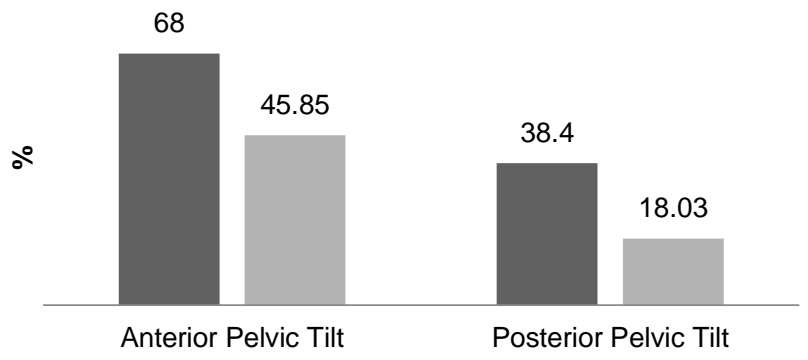

Fig. 8. Percentage of change registered by each of the devices in the two modified tests.

\section{2) Results Corresponding to the Estimated Variance for each Experimental Condition}

Table V shows the corresponding results to the descriptive statistics for each of the sensors and each of the experimental condition assessed.

TABLE V

DESCRIPTIVE STATISTICS CORRESPONDING TO EACH OF THE CORSET TESTS PERFORMED

\begin{tabular}{cccccccc} 
Sensor & Cond. & Min. & Max. & Mean & MSE & SD & Var. \\
\hline $\mathbf{1}$ & & -112.8 & -95.5 & -101.889 & 0.239 & 4.487 & 20.129 \\
$\mathbf{2}$ & & -102.6 & -83.1 & -94.785 & 0.274 & 5.148 & 26.497 \\
$\mathbf{3}$ & $\mathbf{N P}$ & -98.0 & -80.4 & -88.698 & 0.228 & 4.271 & 18.243 \\
$\mathbf{4}$ & & -91.3 & -78.4 & -81.920 & 0.163 & 3.056 & 9.338 \\
$\mathbf{5}$ & & -92.5 & -79.1 & -83.660 & 0.169 & 3.163 & 10.005 \\
$\mathbf{1}$ & & -127.6 & -105.2 & -116.145 & 0.381 & 7.076 & 50.066 \\
$\mathbf{2}$ & & -125.2 & -92.3 & -109.442 & 0.506 & 9.401 & 88.378 \\
$\mathbf{3}$ & $\mathbf{A P T}$ & -111 & -84 & -98.65 & 0.306 & 5.692 & 32.398 \\
$\mathbf{4}$ & & -96.4 & -78.0 & -85.717 & 0.230 & 4.265 & 18.192 \\
$\mathbf{5}$ & & -96.7 & -72.3 & -84.112 & 0.312 & 5.800 & 33.642 \\
$\mathbf{1}$ & & -117.6 & -87.5 & -100.081 & 0.383 & 6.844 & 46.841 \\
$\mathbf{2}$ & & -98.4 & -84.4 & -92.541 & 0.141 & 2.520 & 6.351 \\
$\mathbf{3}$ & $\mathbf{P P T}$ & -103.6 & -82.8 & -91.915 & 0.252 & 4.500 & 20.248 \\
$\mathbf{4}$ & & -95.1 & -78.5 & -86.323 & 0.250 & 4.474 & 20.019 \\
$\mathbf{5}$ & & -100.1 & -78.4 & -87.751 & 0.255 & 4.554 & 20.741 \\
\hline
\end{tabular}

Cond. $=$ Condition; NP=Neutral Position; APT $=$ Anterior Pelvic Tilt; PPT= Posterior Pelvic Tilt; Min=Minimum; Max=Maximum; MSE=Mean Standard Error; $\mathrm{SD}=$ Standard Deviation; Var=Variance 
Table VI shows the results of the comparison between the different tests showing significant differences in all the sensors among the three conditions evaluated. The most marked differences come from the anterior pelvic tilt condition regarding to the neutral and the posterior tilt conditions. The sensors with greater changes are mainly the sensors 1 and 2 .

TABLE VI

Multiple Comparisons ANOVA (one-way)

\begin{tabular}{|c|c|c|c|c|c|c|}
\hline \multirow{2}{*}{ Sensor } & \multirow{2}{*}{\multicolumn{2}{|c|}{ Condition }} & \multirow{2}{*}{ MD } & \multirow{2}{*}{ MSE } & \multicolumn{2}{|c|}{$95 \%$ CI } \\
\hline & & & & & LL & UL \\
\hline \multirow{3}{*}{1} & \multirow{2}{*}{ Neutral } & APT & $14.376^{*}$ & 0.715 & 12.973 & 15.779 \\
\hline & & PPT & $-1.354^{*}$ & 0.518 & -2.371 & -0.338 \\
\hline & APT & PPT & $-15.730^{*}$ & 0.693 & -17.090 & -14.370 \\
\hline \multirow{3}{*}{2} & \multirow{2}{*}{ Neutral } & APT & $13.528^{*}$ & 0.610 & 12.329 & 14.726 \\
\hline & & PPT & $-2.702^{*}$ & 0.442 & -3.570 & -1.834 \\
\hline & $\mathrm{APT}$ & PPT & $-16.229^{*}$ & 0.592 & -17.391 & -15.068 \\
\hline \multirow{3}{*}{3} & \multirow{2}{*}{ Neutral } & $\mathrm{APT}$ & $9.470^{*}$ & 0.447 & 8.592 & 10.348 \\
\hline & & PPT & $3.937^{*}$ & 0.324 & 3.301 & 4.573 \\
\hline & $\mathrm{APT}$ & PPT & $-5.533^{*}$ & 0.434 & -6.385 & -4.682 \\
\hline \multirow{3}{*}{4} & \multirow{2}{*}{ Neutral } & $\mathrm{APT}$ & $4.508^{*}$ & 0.389 & 3.745 & 5.271 \\
\hline & & PPT & $5.405^{*}$ & 0.282 & 4.852 & 5.958 \\
\hline & APT & PPT & $0.897^{*}$ & 0.377 & 0.157 & 1.637 \\
\hline \multirow{3}{*}{5} & \multirow{2}{*}{ Neutral } & APT & $3.469^{*}$ & 0.437 & 2.612 & 4.327 \\
\hline & & PPT & $4.540^{*}$ & 0.316 & 3.919 & 5.161 \\
\hline & $\mathrm{APT}$ & PPT & $1.071^{*}$ & 0.423 & 0.240 & 1.902 \\
\hline
\end{tabular}

MSE=Mean Standard Error; 95\% CI=Confidence Interval; APT=Anterior Pelvic Tilt; PPT=Posterior Pelvic Tilt; $\mathrm{MD}=$ Mean Difference; $\mathrm{LL}=$ Lower limit; UL=Upper Limit.

*Mean Difference is significant at level 0.05

\section{Usability and Comfort Assessment of Lumbatex}

The Table VII and Fig. 12 show the obtained values from participants in the usability and comfort assessment of Lumbatex.

TABLE VII

RESULTS FROM EACH ITEM EVALUATED IN THE COMFORT QUESTIONNAIRE $(\mathrm{N}=15)$

\begin{tabular}{lcccc} 
& Min. & Max. & Mean & SD \\
\hline Waist Fastening & 53 & 147 & 119.27 & 29.224 \\
Spine Fastening & 87 & 150 & 122.87 & 19.820 \\
Tissue & 46 & 150 & 123.67 & 29.083 \\
Compression & 22 & 149 & 129.13 & 31.350 \\
Static comfort & 106 & 150 & 135.67 & 12.910 \\
Dynamic comfort & 78 & 147 & 125.13 & 20.301 \\
Temperature & 53 & 150 & 133.27 & 25.172 \\
Humidity & 53 & 150 & 132.80 & 25.616 \\
Design & 67 & 150 & 122.07 & 24.324 \\
General Comfort & 75 & 146 & 126.87 & 18.201 \\
\hline \multicolumn{4}{c}{ Min=Minimum; Max=Maximum; SD=Standard Deviation }
\end{tabular}

Fig. 9 shows the values (\%) from the subjective perception of the participants about the different categories analysed for assessing the comfort and usability perception. The feeling of comfort presented its best values in the categories of humidity, temperature and static comfort. Otherwise, the lowest value was obtained in the sensation produced by the waist and the spine fastening and also by the design of device. Tissue, movement and compression items were assessed with a mean score. Finally, the overall assessment of the usability and comfort of the device presented high values.

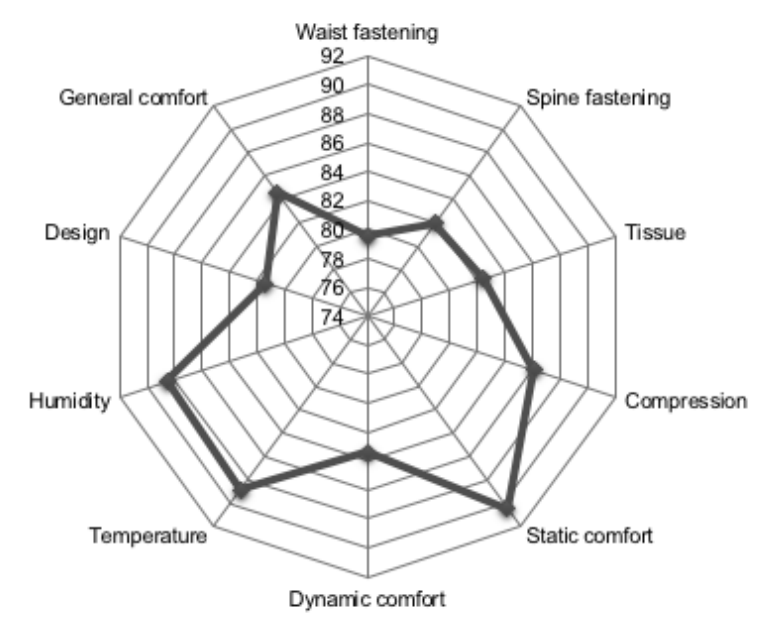
$(\%)$.

Fig. 9. Subjective percentage values of comfort and usability perception

\section{DISCUSSION AND CONCLUSIONS}

The aim of this research was to develop a device based on the integration of wearable MEMS inertial sensor technology in smart textiles, that allows to quantify and measure each segmental vertebral position of lumbar spine. Additionally, it also can monitor the lumbar curvature, providing real-time feedback of all the movements and changes produced in the lordosis of lumbar spine in a sagittal plane. Complementarily, it was proposed to develop a software for obtaining the data from the sensors and interpreting them. Finally, this study aimed to carry out the mechanical and dynamic validation of the system and the usability and comfort assessment of the belt.

The results of testing procedures showed that the Lumbatex system could be used to objectively measure, quantify, reproduce and provide mechanical feedback of the segmental position of lumbar spine. Also, this wearable system could accurately measure the changes on the lumbar lordosis and the movements produced in a sagittal plane. Finally, this device could provide direct and real-time biofeedback for the users of the device. In addition, the good values of the usability and comfort assessment demonstrates that this belt could be easily and snugly used, providing an adequate user's comfort degree. All these features allow that this wearable system could be useful for real-life monitoring of spinal lumbar curvature, out of controlled laboratory environments.

Due to this system presented good results of reliability, validity and comfort, it could potentially be used in medical rehabilitation, sports training and physical therapy, or in daily- 
life activities. The purpose of Lumbatex is to provide to general users with a proper and accurate information about the lumbar spine position and their changes and movements, thereby helping them to avoid incorrect postures or an unsuitable exercise performance.

According to recent research [46], [47] the use of motion capture systems for the quantitative assessment of human movement, using or not wearable sensor-based technology, has been rapidly growing in last years. These systems comprise a series of optoelectronic-based systems, motion capture systems, computer-assisted electromechanical devices, etc. Often, they use accelerometers, electronic goniometers, radiographic assessment or strain-gauge based technology for measure or calculate joint angles, ROM or the back shape. However, some limitations still exist in these systems for the assessment of kinematics and kinetics of lumbar spine. This is due to most of the existent devices cannot measure real-time spine kinematics and monitor changes at segmental level [33], [37], [38], [39], [40], [41]; or do not provide to the user with real-time biofeedback [33], [34]; or they are not wearable devices [34], [35], limiting the data collection to laboratory environments [34], [35], [39]. However, Lumbatex can cover these demands, offering a low-cost, simplified and valid system of data processing, with comfortable fixation method and good batteries live. These features enable that Lumbatex can replace the traditional systems allowing data collection outside laboratory settings. Therefore, this device presents as a practical choice for in-field measurements, thereby enabling analysis in real-life settings where movement disorders may develop and perpetuate, as in the case of LBP [3], [5], [44].

In conclusion, it is important to emphasize the use of Lumbatex as a key strategy for kinematic monitoring of the lumbar spine at a segmental level. In this sense, this device could optimize the body posture and help to prevent or reduce the incidence of mechanical back injuries as well as the LBP. As it has already been documented in this study, LBP is a serious health problem that affects the entire population worldwide. In fact, LBP is currently the most common musculoskeletal pathology within primary care [5], [6], [7]. At present, there are very few known options that offer effective and satisfactory treatment as a solution to this global health problem and the consequence is a considerable health and social expenditure [7], [45].

Given that it is well documented in scientific research that uncontrolled movements and incorrect body postures are the major risk factors in the onset of LBP [44], it currently seems necessary, timely and congruent the development of lumbar monitoring systems. Subsequently, Lumbatex is a new device that offers real-time biofeedback and allows an adequate segmental monitoring of the vertebral neutral zone. This system provides a correct monitoring of the lumbar spine motion, causing the adoption of safe body postures and proper movement performances. In addition, the integration of the system into smart textiles makes the device easily wearable, providing a greater comfort to the final user. Therefore, Lumbatex offers promising practical application possibilities, not only in the clinical and health fields, but also in the field of daily-life ergonomics and also in the different fields of physical activity and sport.
Finally, it is remarkable that, although Lumbatex system comprises 7 sensors, only the five centrals have been evaluated in this study, assessing their capacity to measure and quantify movements and segmental changes of lumbar lordosis in a flexion-extension range. However, other multiplanar measurements could be potentially performed using the present device. Thus, more prospective research should carry out aiming to evaluate the validity and reliability of the system in the measurement and monitoring of multiplanar movements, comprising both lateral bending and rotation motion of the lumbar spine.

\section{REFERENCES}

[1] R. Waldrop, J. Cheng, C. Devin, M. McGirt, M. Fehlings and S. Berven, "The Burden of Spinal Disorders in the Elderly", Neurosurgery, vol. 77, pp. S46-S50, 2015.

[2] A. Alshami, "Prevalence of spinal disorders and their relationships with age and gender", Saudi Medical Journal, vol. 36, no. 6, pp. 725-730, 2015.

[3] R. Horton, "GBD 2010: understanding disease, injury, and risk", The Lancet, vol. 380, no. 9859, pp. 2053-2054, 2012.

[4] S. Indrakanti, M. Weber, S. Takemoto, S. Hu, D. Polly and S. Berven, "Value-based Care in the Management of Spinal Disorders: A Systematic Review of Cost-utility Analysis", Clinical Orthopaedics and Related Research $囚$, vol. 470, no. 4, pp. 1106-1123, 2011.

[5] D. Hoy, C. Bain, G. Williams, L. March, P. Brooks, F. Blyth, A. Woolf, T. Vos and R. Buchbinder, "A systematic review of the global prevalence of low back pain", Arthritis \& Rheumatism, vol. 64, no. 6, pp. 20282037, 2012.

[6] F. Balagué, A. Mannion, F. Pellisé and C. Cedraschi, "Non-specific low back pain", The Lancet, vol. 379, no. 9814, pp. 482-491, 2012.

[7] L. Andersen, T. Clausen, H. Burr and A. Holtermann, "Threshold of Musculoskeletal Pain Intensity for Increased Risk of Long-Term Sickness Absence among Female Healthcare Workers in Eldercare", PLOS ONE, vol. 7, no. 7, p. e41287, 2012.

[8] S. Negrini, G. Imperio, J. Villafañe, F. Negrini and F. Zaina, "Systematic reviews of physical and rehabilitation medicine Cochrane contents. Part 1. Disabilities due to spinal disorders and pain syndromes in adults.", JournalEuropean Journal of Physical and Rehabilitation Medicine, vol. 49, no. 4, pp. 597-610, 2013.

[9] R. Lomas-Vega, M. Garrido-Jaut, A. Rus and R. del-Pino-Casado, "Effectiveness of Global Postural Re-education for Treatment of Spinal Disorders", American Journal of Physical Medicine \& Rehabilitation, vol. 96, no. 2, pp. 124-130, 2017.

[10] X. Wang, J. Zheng, Z. Yu, X. Bi, S. Lou, J. Liu, B. Cai, Y. Hua, M. Wu, M. Wei, H. Shen, Y. Chen, Y. Pan, G. Xu and P. Chen, "A Meta-Analysis of Core Stability Exercise versus General Exercise for Chronic Low Back Pain", PLoS ONE, vol. 7, no. 12, p. e52082, 2012.

[11] R. Caffaro, F. França, T. Burke, M. Magalhães, L. Ramos and A. Marques, "Postural control in individuals with and without non-specific chronic low back pain: a preliminary case-control study", European Spine Journal, vol. 23, no. 4, pp. 807-813, 2014.

[12] J. Hayden, M. van Tulder and G. Tomlinson, "Systematic Review: Strategies for Using Exercise Therapy to Improve Outcomes in Chronic Low Back Pain", Annals of Internal Medicine, vol. 142, no. 9, p. 776, 2005.

[13] J. Hayden, M. van Tulder, A. Malmivaara and B. Koes, "Meta-Analysis: Exercise Therapy for Nonspecific Low Back Pain", Annals of Internal Medicine, vol. 142, no. 9, p. 765, 2005.

[14] A. Searle, M. Spink, A. Ho and V. Chuter, "Exercise interventions for the treatment of chronic low back pain: a systematic review and metaanalysis of randomised controlled trials", Clinical Rehabilitation, vol. 29, no. 12, pp. 1155-1167, 2015.

[15] D. Steffens, C. Maher, L. Pereira, M. Stevens, V. Oliveira, M. Chapple, L. Teixeira-Salmela and M. Hancock, "Prevention of Low Back Pain", JAMA Internal Medicine, vol. 176, no. 2, p. 199, 2016.

[16] S. McGill, "The biomechanics of low back injury: Implications on current practice in industry and the clinic", Journal of Biomechanics, vol. 30, no. 5, pp. 465-475, 1997. 
[17] S. McGill and V. Kippers, "Transfer of Loads Between Lumbar Tissues During the Flexion-Relaxation Phenomenon", Spine, vol. 19, no. 19, pp. 2190-2196, 1994.

[18] P. Dolan and M. Adams, "Influence of lumbar and hip mobility on the bending stresses acting on the lumbar spine", Clinical Biomechanics, vol. 8, no. 4, pp. 185-192, 1993.

[19] J. Cholewicki, M. Panjabi and A. Khachatryan, "Stabilizing Function of Trunk Flexor-Extensor Muscles Around a Neutral Spine Posture", Spine, vol. 22, no. 19, pp. 2207-2212, 1997.

[20] S. McGill, Low back disorders. Champaign: Human Kinetics, 2016.

[21] J. Suni, M. Rinne, A. Natri, M. Statistisian, J. Parkkari and H. Alaranta, "Control of the Lumbar Neutral Zone Decreases Low Back Pain and Improves Self-Evaluated Work Ability", Spine, vol. 31, no. 18, pp. E611E620, 2006.

[22] R. Rondinelli, E. Genovese, R. Katz, T. Mayer, K. Mueller, M. Ranavaya and C. Brigham, Guides to the evaluation of permanent impairment. Chicago, Ill.: American Medical Association, 2009.

[23] R. Lee, J. Laprade and E. Fung, "A real-time gyroscopic system for three-dimensional measurement of lumbar spine motion", Medical Engineering \& Physics, vol. 25, no. 10, pp. 817-824, 2003.

[24] J. Williams, I. Haq and R. Lee, "A novel approach to the clinical evaluation of differential kinematics of the lumbar spine", Manual Therapy, vol. 18, no. 2, pp. 130-135, 2013.

[25] C. Duc, P. Salvia, A. Lubansu, V. Feipel and K. Aminian, "A wearable inertial system to assess the cervical spine mobility: Comparison with an optoelectronic-based motion capture evaluation", Medical Engineering \& Physics, vol. 36, no. 1, pp. 49-56, 2014.

[26] S. McGill, R. Hughson and K. Parks, "Changes in lumbar lordosis modify the role of the extensor muscles", Clinical Biomechanics, vol. 15, no. 10, pp. 777-780, 2000.

[27] J. Oh, H. Cynn, J. Won, O. Kwon and C. Yi, "Effects of Performing an Abdominal Drawing-in Maneuver During Prone Hip Extension Exercises on Hip and Back Extensor Muscle Activity and Amount of Anterior Pelvic Tilt", Journal of Orthopaedic \& Sports Physical Therapy, vol. 37, no. 6, pp. 320-324, 2007.

[28] M. Panjabi, "Clinical spinal instability and low back pain", Journal of Electromyography and Kinesiology, vol. 13, no. 4, pp. 371-379, 2003.

[29] K. Saber-Sheikh, E. Bryant, C. Glazzard, A. Hamel and R. Lee, "Feasibility of using inertial sensors to assess human movement", Manual Therapy, vol. 15, no. 1, pp. 122-125, 2010.

[30] K. Aminian, "Human movement capture and their clinical applications", in Computational intelligence for movement sciences: neural networks, support vector machines and other emerging techniques, 1 st ed., R. Begg and M. Palaniswami, Ed. USA: Idea Group Inc., 2006, pp. 101-138.

[31] H. Zhou, T. Stone, H. Hu and N. Harris, "Use of multiple wearable inertial sensors in upper limb motion tracking", Medical Engineering \& Physics, vol. 30, no. 1, pp. 123-133, 2008.

[32] A. Cuesta-Vargas, A. Galán-Mercant and J. Williams, "The use of inertial sensors system for human motion analysis", Physical Therapy Reviews, vol. 15, no. 6, pp. 462-473, 2010.

[33] A. Mannion, K. Knecht, G. Balaban, J. Dvorak and D. Grob, "A new skin-surface device for measuring the curvature and global and segmental ranges of motion of the spine: reliability of measurements and comparison with data reviewed from the literature", European Spine Journal, vol. 13, no. 2, pp. 122-136, 2004.

[34] A. Ranavolo, R. Don, F. Draicchio, M. Bartolo, M. Serrao, L. Padua, G. Cipolla, F. Pierelli, S. Iavicoli and G. Sandrini, "Modelling the spine as a deformable body: Feasibility of reconstruction using an optoelectronic system", Applied Ergonomics, vol. 44, no. 2, pp. 192-199, 2013.

[35] S. Schmid, D. Studer, C. Hasler, J. Romkes, W. Taylor, S. Lorenzetti and R. Brunner, "Quantifying spinal gait kinematics using an enhanced optical motion capture approach in adolescent idiopathic scoliosis", Gait \& Posture, vol. 44, pp. 231-237, 2016.

[36] T. Consmüller, A. Rohlmann, D. Weinland, C. Druschel, G. Duda and W. Taylor, "Comparative evaluation of a novel measurement tool to assess lumbar spine posture and range of motion", European Spine Journal, vol. 21, no. 11, pp. 2170-2180, 2012.

[37] W. Taylor, T. Consmüller and A. Rohlmann, "A novel system for the dynamic assessment of back shape", Medical Engineering \& Physics, vol. 32, no. 9, pp. 1080-1083, 2010.

[38] K. O'Sullivan, L. O'Sullivan, A. Campbell, P. O'Sullivan and W. Dankaerts, "Towards monitoring lumbo-pelvic posture in real-life situations: Concurrent validity of a novel posture monitor and a traditional laboratory-based motion analysis system", Manual Therapy, vol. 17, no. 1, pp. 77-83, 2012.
[39] R. Mieritz, G. Bronfort, M. Jakobsen, P. Aagaard and J. Hartvigsen, "Reliability and measurement error of sagittal spinal motion parameters in 220 patients with chronic low back pain using a three-dimensional measurement device", The Spine Journal, vol. 14, no. 9, pp. 1835-1843, 2014.

[40] C. Goodvin, E. Park, K. Huang and K. Sakaki, "Development of a realtime three-dimensional spinal motion measurement system for clinical practice", Medical \& Biological Engineering \& Computing, vol. 44, no. 12, pp. 1061-1075, 2006.

[41] J. Lee, G. Desmoulin, A. Khan and E. Park, "Comparison of 3D spinal motions during stair-climbing between individuals with and without low back pain", Gait \& Posture, vol. 34, no. 2, pp. 222-226, 2011.

[42] C. Richardson, "The time to move forward", in Therapeutic Exercise for Lumbopelvic Stabilization, 2nd ed., C. Richardson, P. Hodges and J. Hides, Brisbane Ed.: Churchill Livingstone, 2018, p. 280.

[43] A. Lucas-Cuevas, P. Pérez-Soriano, J. Priego-Quesada and S. LlanaBelloch, "Influence of foot orthosis customization on perceived comfort during running", Ergonomics, vol. 57, no. 10, pp. 1590-1596, 2014.

[44] W. Hoogendoorn, P. Bongers, H. de Vet, M. Douwes, B. Koes, M. Miedema, G. Ariëns and L. Bouter, "Flexion and Rotation of the Trunk and Lifting at Work Are Risk Factors for Low Back Pain", Spine, vol. 25, no. 23, pp. 3087-3092, 2000.

[45] C. Williams, C. Maher, M. Hancock, J. McAuley, A. McLachlan, H. Britt, S. Fahridin, C. Harrison, J. Latimer, "Low Back Pain and Best Practice Care", Archives of Internal Medicine, vol. 170, no. 3, p. 271, 2010

[46] E. Papi, W. Koh and A. McGregor, "Wearable technology for spine movement assessment: A systematic review", Journal of Biomechanics, vol. 64, pp. 186-197, 2017.

[47] Fong and Y. Chan, "The Use of Wearable Inertial Motion Sensors in Human Lower Limb Biomechanics Studies: A Systematic Review", Sensors, 10(12), 11556-11565, 2019. 